\title{
Stochastic BEM for the Vibroacoustic Analysis of Three-Dimensional Structures
}

\author{
R. D’Amico, A. Pratellesi, M. Pierini, and N. Baldanzini \\ Dipartimento di Meccanica e Tecnologie Industriali, Università degli Studi di Firenze, Via di Santa Marta 3, \\ 50139 Firenze, Italy
}

Correspondence should be addressed to R. D’Amico, roberto.damico@unifi.it

Received 14 January 2011; Revised 24 April 2011; Accepted 24 May 2011

Academic Editor: Kok Keong Choong

Copyright ( 2011 R. D’Amico et al. This is an open access article distributed under the Creative Commons Attribution License, which permits unrestricted use, distribution, and reproduction in any medium, provided the original work is properly cited.

\begin{abstract}
Nowadays, extending the NVH prediction reliability to the whole frequency range is an attractive goal of vibroacoustics. Deterministic methodologies are well established for the low-frequency range, but, decreasing the wavelength, energy-based methods are necessary. In such a range, a crucial role is played by small perturbations which highly influence the response sensitivity. Moreover, taking into account these variations allows to make the product design more robust and even quicker. Introducing geometrical uncertainties within the classic BEM formulation allows to obtain the so-called stochastic BEM. As a result, the solution shows deterministic behaviour at low frequencies; decreasing the wavelength, the effect of the uncertainties smooths the response. Consequently, it is possible to obtain an averaged trend over the whole frequency range which asymptotically tends to the deterministic one. In this paper, we deal with three-dimensional acoustic SBEM. First, the formulation and its basic assumptions are presented. Secondly, they are applied to academic cases to show its potentialities in predicting vibroacoustic behaviour over a wide frequency range.
\end{abstract}

\section{Introduction}

To correctly predict the midfrequency behaviour of structures is a crucial objective of vibro-acoustics. Generally, a mechanical structure is a system made by several components with different geometrical and material properties which consequently exhibit different vibro-acoustic behaviours. The prediction becomes even more problematic if uncertainties and perturbations are taken into account in the analysis.

Depending on the structure dimension, the whole frequency range can be split in three domains. The low-frequency domain is characterized by a long wavelength in in respect to the system dimension. In the high-frequency range, the wavelength is much smaller respect to such a dimension. Finally, a transition region exists, the so-called midfrequency range.

Deterministic techniques as finite element method (FEM) [1] and boundary element method (BEM) [2,3] guarantee a reliable prediction at low-frequencies. The current computational resources allow these numerical methods to be efficient even for complex structures as far as the low-frequency domain is concerned. Nevertheless, the smaller the wavelength, the more refined the discretization required and is obtaining an accurate prediction becomes very demanding. However, deterministic methods can be pushed up in frequency by means of stabilization and accelerating techniques, and it becomes possible to use them in the middle range. Fast multipole methods [4] and domain decomposition and stabilized FE [5] are enhancements of, respectively, classic BEM and FEM to shift their usage up to higher frequencies. A novel wave based method (WBM) [6] allows avoiding large computational resources and model discretization.

At low frequencies, material properties and geometry behaviour are known with sufficient precision and the response is not so sensitive to variations. At high-frequencies model properties are highly uncertain, and the solution is very sensitive to small perturbations. Manohar and Keane $[7,8]$ highlight these effects computing the eigenfrequency density functions of a beam whose mass density is affected by random uncertainty. Moreover, according to Fahy and Mohammed [9], the differences among systems, which share the same design characteristics and the effects of these 
differences on vibrational behaviour, are individually unpredictable in the high-frequency range, therefore a probabilistic model is appropriate. The effect of this sensitiveness can be seen in Figure 1. The FRFs of a plane plate, obtained with a Monte Carlo simulation, varying the geometrical and physical parameters (length, thickness, width, Young modulus, and density), show that above $100 \mathrm{~Hz}$ it is no more possible to have a precise and defined response, but a spread of results [10]. The average of the spread can be efficiently represented by a smoothened curve which neglects the local peak but predicts the overall trend. Consequently, using deterministic techniques over the whole frequency range becomes meaningless.

The statistical energy analysis (SEA) [11] is a widely employed tool to solve many acoustic and vibration problems in the high frequency range [12]. For SEA, the power transfer between subsystems is proportional to the difference of the model energy levels in the subsystems. As a hypothesis, the structure has to be nonzero damped, input powers uncorrelated, and the subsystems weakly coupled. Moreover, the modal density of each subsystem has to be high. In addition, even if large literature exists, the determination of coupling loss factors, which regulate the energy exchange between subsystems, and the discretization of systems are still crucial aspects to build a reliable model [13]. Moreover, variations in energy density within the subsystem are not taken in account and it is not possible to control directly the uncertainties on the model. To tackle the first issue, the virtual SEA [14] has been carried out. The novelty of the method consists of the development of a frequency dependent procedure for defining the classic SEA subsystem.

Promising approaches are the wave and finite element method (WFE) [15] and the waveguide finite element method [16] to model the structure dynamics which are piecewise homogeneous or periodic in one or two dimensions or which are axisymmetric. The wave methods allow to reduce considerably the dimension of the problem and thus the effort for calculations.

In the midfrequency range complex structures can be classified as comprised of two classes of subsystems, respectively, exhibiting an LF and a HF behaviour. To deal with the modelling requirements, hybrid approaches, which merge deterministic and energy methods, have been developed. Shorter and Langley [17] developed the a hybrid method to couple FE and SEA. Stiff components are modelled with FE since they have low modal density, flexible components are analysed with SEA since they have high modal density [18].

In the high-frequency range it has been proven that the flow of vibrational energy between weakly coupled subsystems is analogous to the way in which heat flows between two bodies of different temperature in a thermal analysis. Starting from the previous assumption, the Energy Flow Method (EFM) approaches the vibro-acoustic analysis in the HF range using a heat-conduction analogy. The method is derived from a local energy balance leading to a constitutive relationship analogous to the heat conduction equation [19]. Energy variations are smoother than displacement, thus an energy flow approach is more efficient even for high-order modes. Moreover, the numerical cost for solving

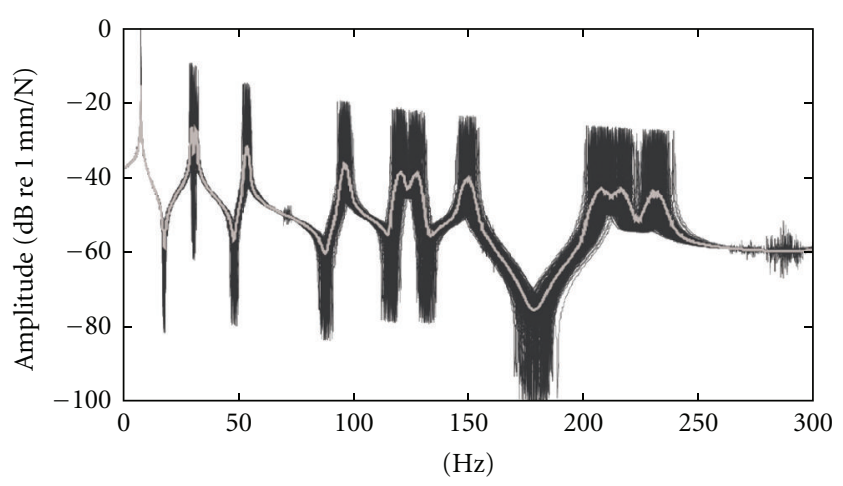

FIGURE 1: From [10], FRFs functions of a plane plate, varying the geometrical and physical parameters. Mean value (grey line) and spread (black).

the thermal problem is reduced compared to the wavebased approach. Many applications of these methods were proposed in the past. In particular, Wohlever, Bouthier, and Bernhard give some results regarding the energy models of rods, Euler-Bernoulli beams [20], membranes [21] and Kirchoff-Love plates [22] applying the power flow analysis. This formulation is obtained computing the real part of the harmonic energy flow balance. The near field contribution is assumed equal to zero, finally the spatial average operation allows obtaining a relation between the total energy density and the active power flow. Lase et al. [23], Ichochou et al. [24] developed the General Energy Method (GEM) for rods and beams. This method consists of expliciting the different terms of the harmonic complex energy flow balance. In this way, energy density, active power flow, Lagrangian density, and reactive power flow are obtained using a wave description. The application of some high-frequency assumptions leads to a simplified energy method (SEM). Lagrangian density and reactive power flow are assumed negligible, since their frequency average are equal to zero. This corresponds to neglecting the evanescent wave field far from the loadings and structure discontinuities, and the interfaces between the propagative waves are not taken for granted.

Solving the problem using these formulations requires the definition of power boundary conditions which are difficult to evaluate. Usually they are replaced by the power associated to infinite or semi-infinite structure. Viktorovitch et al. $[25,26]$ shows that the same power flow analysis and simplifed energy method formulations can be obtained introducing random parameters in the description of the geometrical parameters of the structure. The stochastic approach leads to a smooth response which is strongly influenced by the uncertainty. As a result, the higher the uncertainty, the smoother the response. Moreover, at high frequencies this formulation converges asymptotically to the SEM curve which is the response of the infinite system.

The capability to predict effects of uncertainties also has a direct impact on the product design. Indeed, during the industrialization process, small variations and irregularities are present and influence the vibro-acoustic behaviour of the whole structure, especially at high frequencies. This makes 
the classical deterministic approaches not reliable. Obtaining the average behaviour allows to avoid a demanding Monte Carlo analysis or other sampling procedure, but still to deliver a robust design.

Viktorovitch et al. [27, 28] also introduced the socalled Smooth Integral Formulation (SIF) for one- and two-dimensional cases. This approach leads to a boundary integral formulation coupled with a statistical approach to account for uncertainties in the structural parameters. Introducing randomness to the geometrical or/and material properties of the structure leads to a precise description of the deterministic low frequency response and a smooth response in the high frequency field. This corresponds to the average of the strongly oscillating vibratory response which is solved in one go instead of using a sampling procedure. The starting point for SIF is the direct BEM formulation with the addition of supplementary equations which represent the energetic part of the problem. Differently from the well-known statistical methods, SIF allows to directly control the uncertainties on the model shape. As a result, when the frequency increases and the deterministic solution of the nominal structure starts to be meaningless, the SIF response allows to take into account effects of uncertainties presenting a smoothing behaviour towards an energetic description. On the other hand, if we want to use the energy methods in the midfrequency range, their assumptions become no longer valid. On the contrary, the SIF provides an averaged behaviour of the perturbed system. Since the stochastic approach is applied to a standard BEM formulation, the method is called stochastic boundary element method (SBEM).

Pratellesi et al. [29, 30] and Viktorovitch and Pratellesi [31] developed a hybrid formulation to couple SBEM, employed for the high-frequency part, with the finite element description of the low frequency behaving subsystems. The coupling allows to account for both deterministic and statistical contributions in the response of the structure and therefore to obtain a consistent formulation for the midfrequency range. Application cases were extended to one and two dimensions.

In this paper, SBEM methodology is applied to threedimensional vibro-acoustic cases. First of all, a description of the methodology is presented. Uncertainties are applied to the expectation of the classic BEM formulation. Successively, additional relations are used to model the energetic part of the system. To prove the applicability of SBEM, a rectangular and a spherical acoustic cavity, with different degree of uncertainty, are investigated using SBEM. Results are shown both as deformed shape of a field mesh plane and response function computed at a solution point. Finally, conclusions are drawn, and further steps in research are highlighted.

\section{The Boundary Element Method}

Many problems related to steady-state oscillations lead to the Helmholtz equation,

$$
\nabla^{2} p(\mathbf{x})+k^{2} p(\mathbf{x})=0 \quad \text { on } D
$$

where $p$ is the acoustic pressure at $\mathbf{x}, k$ is the wavenumber $\omega / c, \omega$ is the circular frequency, $c$ is the speed of sound and $D$ is the domain. The boundary element method allows to find an approximate solution to the problem in (1) with proper boundary conditions. In order to obtain the boundary integral formulation, (1) is integrated twice via Green's theorem over one side of the domain, using free space Green's functions

$$
G(\mathbf{x}, \mathbf{y})=\frac{e^{-i k r}}{4 \pi r}
$$

where $r=|\mathbf{x}-\mathbf{y}|$. This leads to the Helmholtz integral equation

$$
\begin{aligned}
c_{s}(\mathbf{x}) p(\mathbf{x})= & \int_{D_{f}} f(\mathbf{y}) G(\mathbf{x}, \mathbf{y}) d V \\
& +\int_{\partial D} p(\mathbf{y}) d G(\mathbf{x}, \mathbf{y}) d s-\int_{\partial D} G(\mathbf{x}, \mathbf{y}) d p(\mathbf{y}) d s,
\end{aligned}
$$

denoting with $d p$ the derivative of the pressure $p$ and $d G$ the derivative of the Green kernel, both with respect to the variable x. $D_{f}$ is the domain in which $f$ is defined. Moreover, $c_{s}(\mathbf{x})$ is a coefficient dependent on the position of the point $\mathbf{x}$. If $\mathbf{x}$ is inside the domain $c_{s}$ is equal to 1 , if it is outside the domain $c_{s}$ is equal to 0 , and if it is on an approximately smooth boundary $\partial D, c_{s}$ is equal to $1 / 2$. Let us apply on $\partial D_{p}$ pressure boundary condition

$$
p(\mathbf{x})=\hat{p}(\mathbf{x}) \quad \text { on } \partial D_{p},
$$

and velocity boundary condition on $\partial D_{v}$

$$
d p(\mathbf{x})=\hat{d} p(\mathbf{x}) \quad \text { on } \partial D_{v}
$$

$\partial D_{p}$ and $\partial D_{v}$ constitute partitions of $\partial D$. A proper discretization of the boundary allows to write the fundamental BEM equation

$$
\begin{aligned}
c_{s} p(\mathbf{x})= & \int_{D_{f}} f(\mathbf{y}) G(\mathbf{x}, \mathbf{y}) d V \\
& +\sum_{j=1}^{N_{v}} \int_{\partial D_{v}}\left[p_{j} d G(\mathbf{x}, \mathbf{y})-d \hat{p}_{j} G(\mathbf{x}, \mathbf{y})\right] d s \\
& +\sum_{k=1}^{N_{p}} \int_{\partial D_{p}}\left[\hat{p}_{k} d G(\mathbf{x}, \mathbf{y})-d p_{k} G(\mathbf{x}, \mathbf{y})\right] d s .
\end{aligned}
$$

Firstly the unknowns are computed at the nodes of the boundary, secondly the response is evaluated projecting these boundary contributions at a field solution point.

\section{The Stochastic Boundary Element Method}

3.1. Overview of the Random Formulation. Classic BEM provides a deterministic and reliable prediction in a lowfrequency range. Decreasing the wavelength, sensitivity to small perturbation becomes higher and BEM fails to provide a useful representation of the vibro-acoustic phenomena. 
The SBEM formulation allows to obtain an averaged behaviour over the whole frequency range. Indeed, the prediction is deterministic at low frequencies, where effect of perturbations are negligible. Increasing the frequency, the SBEM takes into account uncertainties achieving a smoothened response.

Many kinds of uncertainties can affect the structure characteristics. In this work we deal with geometrical ones, thus we suppose that the shape of the model and excitation points are perturbed. This is due to the fact that introducing other kind of uncertainties would produce a formulation much more difficult to handle.

Randomized boundary parameters and force application points are expressed as follows

$$
\tilde{x}_{i}=x_{i}+\epsilon_{i}
$$

where $x_{i}$ is the deterministic value of the parameter while $\epsilon_{i}$ is the zero mean random variable. To solve the formulation, a statistic probability distribution should be introduced. It can assume different shapes, Gaussian, triangular, rectangular, hyperbolic, and so forth. The overall density function of the random variables can be evaluated for $n$ independent random variables as

$$
f_{\epsilon_{1}, \ldots, \epsilon_{n}}\left(y_{1}, \ldots, y_{n}\right)=\prod_{i=1}^{n} f_{\epsilon_{i}}\left(y_{i}\right)
$$

The expectation of a generic function of $n$ variables $h\left(\tilde{y}_{1}, \ldots, \tilde{y}_{n}\right)$, where each variable $y_{i}$ has a distribution $f\left(y_{i}\right)$, is

$$
\left\langle h\left(\tilde{y}_{1}, \ldots, \tilde{y}_{n}\right)\right\rangle=\int_{-\infty}^{+\infty} \cdots \int_{-\infty}^{+\infty} h\left(y_{1}, \ldots, y_{n}\right) \prod_{i=1}^{n} f_{\epsilon_{i}}\left(y_{i}\right) d s .
$$

Directly computing the expectation of the boundary element formulation (6), we can evaluate the first-order moment (FOM) of the variable. This quantity does not give interesting information about the system behaviour because increasing the frequency, the first order moment vanishes to zero. Multiplying FOM equations by well-chosen variables, it is possible to obtain the second order Moments of the unknowns. As we previously said, energy variations are smoother than displacement and using an energy flow to evaluate the response is more efficient. Since the second order moments are quantities strictly related to an energy description of the vibrational behaviour, they do not converge to zero but, as the frequency increases, give a smooth trend. Moreover, the high the uncertainty level, the smoother the prediction.

In order to obtain the second order moments of the variables, let us consider (6) computed at point $\widetilde{\mathbf{x}}_{i} \in \partial \widetilde{\Omega}_{v}$, multiply it by the complex conjugate of the unknown variable $\tilde{p}_{i}^{*}$ and finally compute the expectation of the product. Using the linearity property of the expectation operator, we obtain (10) expression

$$
\begin{aligned}
\frac{1}{2}\left\langle\left|\tilde{p}_{i}\right|^{2}\right\rangle= & \left\langle\tilde{p}_{i}^{*} \int_{\tilde{D}_{f}} f(\mathbf{y}) G\left(\mathbf{y}, \tilde{\mathbf{x}}_{i}\right) d V\right\rangle \\
& +\left\langle\tilde{p}_{i}^{*} \sum_{\substack{j=1 \\
j \neq i}}^{N_{v}} \tilde{p}_{j} \int_{\partial \tilde{D}_{j}} d G\left(\mathbf{y}, \tilde{\mathbf{x}}_{i}\right) d s\right\rangle \\
& -\left\langle\tilde{p}_{i}^{*} \sum_{j=1}^{N_{v}} \int_{\partial \tilde{D}_{j}} \hat{d}_{j} G\left(\mathbf{y}, \tilde{\mathbf{x}}_{i}\right) d s\right\rangle \\
& +\left\langle\tilde{p}_{i}^{*} \sum_{k=1}^{N_{p}} \int_{\partial \tilde{D}_{k}} \hat{p}_{k} d G\left(\mathbf{y}, \tilde{\mathbf{x}}_{i}\right) d s\right\rangle \\
& -\left\langle\tilde{p}_{i}^{*} \sum_{k=1}^{N_{p}} \tilde{d}_{p_{k}} \int_{\partial \tilde{D}_{k}} G\left(\mathbf{y}, \tilde{\mathbf{x}}_{i}\right) d s\right\rangle \\
& +\left\langle\left|\tilde{p}_{i}\right|^{2} \int_{\partial \tilde{D}_{i}} d G\left(\mathbf{y}, \tilde{\mathbf{x}}_{i}\right) d s\right\rangle .
\end{aligned}
$$

On the other hand, we can consider (6) computed at point $\tilde{\mathbf{x}}_{i} \in \partial \widetilde{\Omega}_{p}$, multiply it by the complex conjugate of the unknown variable $\tilde{d} p_{i}^{*}$ and finally compute the expectation of the product

$$
\begin{aligned}
\frac{1}{2}\left\langle\left|\tilde{d} p_{i}\right|\right\rangle \hat{p}_{i}= & \left\langle\tilde{d} p_{i}^{*} \int_{\tilde{D}_{f}} f(\mathbf{y}) G\left(\mathbf{y}, \tilde{\mathbf{x}}_{i}\right) d V\right\rangle \\
& +\left\langle\tilde{d} p_{i}^{*} \sum_{j=1}^{N_{v}} \tilde{p}_{j} \int_{\partial \tilde{D}_{j}} d G\left(\mathbf{y}, \tilde{\mathbf{x}}_{i}\right) d s\right\rangle \\
& -\left\langle\tilde{d} p_{i}^{*} \sum_{j=1}^{N_{v}} \int_{\partial \tilde{D}_{j}} \hat{d} p_{j} G\left(\mathbf{y}, \tilde{\mathbf{x}}_{i}\right) d s\right\rangle \\
& +\left\langle\tilde{d} p_{i}^{*} \sum_{k=1}^{N_{p}} \int_{\partial \tilde{D}_{k}} \hat{p}_{k} d G\left(\mathbf{y}, \tilde{\mathbf{x}}_{i}\right) d s\right\rangle \\
& -\left\langle\tilde{d} p_{i}^{*} \sum_{\substack{N_{p} \\
k \neq i}} \tilde{d}_{p_{k}} \int_{\partial \tilde{D}_{k}} d G\left(\mathbf{y}, \tilde{\mathbf{x}}_{i}\right) d s\right\rangle \\
& +\left\langle\left|\tilde{d}_{p_{i}}\right|^{2} \int_{\partial \tilde{D}_{i}} d G\left(\mathbf{y}, \tilde{\mathbf{x}}_{i}\right) d s\right\rangle
\end{aligned}
$$

Equations (10) and (11) contain high-order statistical moments and a large number of unknowns. Since the problem has much more unknowns than equations, cross-products have to be simplified and some statistical assumptions have to be introduced.

3.2. Assumptions. To reduce the number of unknown crossproducts and the amount of supplementary equations needed to solve the problem, we introduce three assumptions based on physical considerations. 
The first two assumptions are related to isolated systems and define the dependence between contributions coming from different sources. On the other hand, considering systems which mutually exchange power, a third assumption for nonisolated structures has to be used. This assumption defines the coupling condition between two or more systems and allows to model the power exchange from one to the other.

To introduce the assumptions, we define two kind of sources: power inputs as external loadings or velocity boundary conditions are considered as primary sources; boundary conditions which not describe a power transfer are named secondary sources. The latter are constituted by the multiple wave reflections of the wave stemming from the loadings.

Assumption 1. The contributions of two sources are statistically independent when the positions of the sources or the target points are distinct. The physical reasons behind this assumption are the following: positions of different sources and target points are statistically independent because the two contributions can be supposed independent; each unknown is naturally correlated only to the power inputs and then to loadings and velocity boundary conditions.

Assumption 2. It is considered that a force or a displacement variable expressed at any point of the structure is only correlated with the contribution of the primary sources at that point. This assumption states that Green's function and the related intensity of a secondary source are independent and the average of the product is equal to the product of the averages. It is important to explain the physical meaning of this assumption. Let us consider two frequency-dependent functions A and B. Each of them can be expressed as

$$
\begin{aligned}
& A(\omega)=\langle A(\omega)\rangle+\epsilon_{A}(\omega), \\
& B(\omega)=\langle B(\omega)\rangle+\epsilon_{B}(\omega),
\end{aligned}
$$

where the average of the fluctuation $\epsilon$ is equal to zero. If we compute the expectation of the product, we obtain

$$
\langle A(\omega) B(\omega)\rangle=\langle A(\omega)\rangle\langle B(\omega)\rangle+\left\langle\epsilon_{A}(\omega) \epsilon_{B}(\omega)\right\rangle .
$$

The cross-products are suppressed form (13) because they are equal to zero since they are defined as fluctuating functions. We can discuss the physical reasons which cause the vanishing of the second term on the right-hand side. The convergence towards zero depends on their correlation, amplitude, and frequency of oscillations. Let us consider the Green functions $G\left(x, y_{1}\right)$ and $G\left(x, y_{2}\right)$ instead of $A$ and $B$. When $y_{1} \approx y_{2}$, the difference of amplitude fluctuations are small and the two functions overlap as frequency increases. On the other hand, when $y_{1} \neq y_{2}, G\left(x, y_{1}\right)$ and $G\left(x, y_{2}\right)$ are different, independent and rapidly oscillate at medium and high frequencies. If $y_{1}$ is far away from $y_{2}$, the randomness introduced at $y_{1}$ it is not directly correlated to the randomness introduced at $y_{2}$. In general, approaching the mid- and high-frequency range the high modal overlap, the high modal density and the low amplitude fluctuations allow to state that the sources are independent.
Assumption 3. The boundaries connecting two substructures, of which one contains a primary source, become primary sources for the other substructure. This assumption allows to model the power flow from one system to the other. Finally, we can state a general rule which can synthesize all of them: unknowns are only dependent on sources which contribute to the power flow within the system.

3.3. Final Formulation. Applying the first two assumptions, the fundamental SBEM equations are obtained. For $\tilde{\mathbf{x}}_{i} \in$ $\partial \widetilde{\Omega}_{v},(10)$ becomes

$$
\begin{aligned}
\frac{1}{2}\left\langle\left|\tilde{p}_{i}\right|^{2}\right\rangle= & \left\langle\tilde{p}_{i}^{*} \int_{\tilde{D}_{f}} f(\mathbf{y}) G\left(\mathbf{y}, \tilde{\mathbf{x}}_{i}\right) d V\right\rangle \\
& +\left\langle\tilde{p}_{i}^{*}\right\rangle \sum_{\substack{j=1 \\
j \neq i}}^{N_{v}}\left\langle\tilde{p}_{j}\right\rangle\left\langle\int_{\partial \tilde{D}_{j}} d G\left(\mathbf{y}, \tilde{\mathbf{x}}_{i}\right) d s\right\rangle \\
& -\left\langle\tilde{p}_{i}^{*}\right\rangle \sum_{j=1}^{N_{v}}\left\langle\int_{\partial \tilde{D}_{j}} \hat{d} p_{j} G\left(\mathbf{y}, \tilde{\mathbf{x}}_{i}\right) d s\right\rangle \\
& +\left\langle\tilde{p}_{i}^{*}\right\rangle \sum_{k=1}^{N_{p}}\left\langle\int_{\partial \widetilde{D}_{k}} \hat{p}_{k} d G\left(\mathbf{y}, \tilde{\mathbf{x}}_{i}\right) d s\right\rangle \\
& -\left\langle\tilde{p}_{i}^{*}\right\rangle \sum_{k=1}^{N_{p}}\left\langle\tilde{d}_{p_{k}}\right\rangle\left\langle\int_{\partial \tilde{D}_{k}} G\left(\mathbf{y}, \tilde{\mathbf{x}}_{i}\right) d s\right\rangle \\
& +\left\langle\left|\tilde{p}_{i}\right|^{2}\right\rangle\left\langle\int_{\partial \tilde{D}_{i}} d G\left(\mathbf{y}, \tilde{\mathbf{x}}_{i}\right) d s\right\rangle .
\end{aligned}
$$

Applying the assumptions to (11) at point $\tilde{\mathbf{x}}_{i} \in \partial \widetilde{\Omega}_{p}$, it becomes

$$
\begin{aligned}
& \frac{1}{2}\left\langle\left|\tilde{d} p_{i}\right|\right\rangle \hat{p}_{i}=\left\langle\tilde{d} p_{i}^{*} \int_{\widetilde{D}_{f}} f(\mathbf{y}) G\left(\mathbf{y}, \widetilde{\mathbf{x}}_{i}\right) d V\right\rangle \\
& +\left\langle\tilde{d}_{i}^{*}\right\rangle \sum_{j=1}^{N_{v}}\left\langle\tilde{p}_{j}\right\rangle\left\langle\int_{\partial \tilde{D}_{j}} d G\left(\mathbf{y}, \tilde{\mathbf{x}}_{i}\right) d s\right\rangle \\
& -\left\langle\tilde{d} p_{i}^{*}\right\rangle \sum_{j=1}^{N_{v}}\left\langle\int_{\partial \widetilde{D}_{j}} \hat{d} p_{j} G\left(\mathbf{y}, \tilde{\mathbf{x}}_{i}\right) d s\right\rangle \\
& +\left\langle\tilde{d} p_{i}^{*}\right\rangle \sum_{k=1}^{N_{p}}\left\langle\int_{\partial \tilde{D}_{k}} \hat{p}_{k} d G\left(\mathbf{y}, \tilde{\mathbf{x}}_{i}\right) d s\right\rangle \\
& -\left\langle\tilde{d} p_{i}^{*}\right\rangle \sum_{\substack{k=1 \\
k \neq i}}^{N_{p}}\left\langle\tilde{d} p_{k}\right\rangle\left\langle\int_{\partial \tilde{D}_{k}} G\left(\mathbf{y}, \tilde{\mathbf{x}}_{i}\right) d s\right\rangle \\
& +\left\langle\left|\tilde{d} p_{i}\right|^{2}\right\rangle\left\langle\int_{\partial \tilde{D}_{i}} d G\left(\mathbf{y}, \tilde{\mathbf{x}}_{i}\right) d s\right\rangle
\end{aligned}
$$


One can observe that the number of unknowns are equal to $3\left(N_{p}+N_{v}\right)$. They are as follows.

(i) First order moments (FOM): $\left\langle\tilde{p}_{i}\right\rangle$ and $\left\langle\tilde{d}_{p_{i}}\right\rangle$.

(ii) Second order moments (SOM): $\left\langle\left|\tilde{p}_{i}\right|^{2}\right\rangle$ and $\left\langle\left|\tilde{d} p_{i}\right|^{2}\right\rangle$.

(iii) Correlated unsplittable products of kinematic variables multiplied by primary source: $\left\langle\tilde{p}_{i}^{*} \int_{\tilde{D}_{f}} f(\mathbf{y}) G(\mathbf{y}\right.$, $\left.\left.\tilde{\mathbf{x}}_{i}\right) d V\right\rangle$ and $\left\langle\tilde{d} p_{i}^{*} \int_{\tilde{D}_{f}} f(\mathbf{y}) G\left(\mathbf{y}, \tilde{\mathbf{x}}_{i}\right) d V\right\rangle$.

To solve the system, other $2\left(N_{p}+N_{v}\right)$ equations have to be added. $\left(N_{p}+N_{v}\right)$ equations are obtained by considering the expectation of the classical boundary integral equations (6). Finally, the last $\left(N_{p}+N_{v}\right)$ equations are obtained by multiplying each side of the conjugate of (6) by the contribution of the external loading. As a result, the problem

$$
A \mathbf{p}=\mathbf{b}
$$

is solved directly: $\mathbf{p}$ is the unknown vector $3\left(N_{p}+N_{v}\right) \times 1$, $A$ is a martix $3\left(N_{p}+N_{v}\right) \times 3\left(N_{p}+N_{v}\right)$, and $\mathbf{b}$ is a vector $3\left(N_{p}+N_{v}\right) \times 1$. Due to the equations, matrix $A$ has sparse form and elements are complex.

After the computation of the unknowns, Second-order Moments are evaluated at the field solution point.

Looking at (14) and (15), we can easily understand that the SBEM code is penalized in the low-frequency and small model applications, but it has some advantages over the classical methods when dealing with high frequencies. Even if the number of equation increases, the mesh coarsening process reduces the computational effort. As a rule of thumb, using standard BEM at least 6 element/wavelength are needed in order to obtain a reliable solution. With SBEM things are slightly different. Increasing the frequency, the wavelength reach the size of the small perturbations and uncertainties start to influence the response. This means that the mesh does not need to be refined to reach higher frequency values as happens for BEM but only to reach frequencies at which the uncertainties start playing their role. Moreover, the SBEM gives a statistical result which gives much more details and robustness than a classical deterministic result. As reported in Section 4, using a few elements allows moving up to ranges where standard BEM fails to provide accurate solutions. Moreover, if compared to classic sampling methods for uncertainties modelling it does not require to calculate and average over a population of structures, since the averaging effect is included in the expected terms. Only one run of the program for each frequency step is necessary to obtain an averaged statistical response. Finally, it allows easy coupling with FEM or BEM $[30,31]$.

It is important to note that boundary conditions play a crucial role as power inputs. Since all the unknowns are dependent on power inputs, a nonzero-velocity boundary condition applied on a large amount of nodes drastically increases the system dimension. Indeed, each node has to be considered as primary source for the system. In this paper we focus our attention only on cases with zero-velocity (or rigid walls) boundary condition.
3.4. Integrals and Integration. In order to solve the SBEM set of equations, the numerical evaluation of the expectations of boundary and domain integrals must be carried out as indicated in (9). The integration path in two- and threedimensional applications is random, therefore it is not possible to commutate the expectation and the integral operators (as it is possible for rods and beams, one-dimensional elements). To simplify this evaluation, it can be shown that the random variable can be judiciously chosen in order to get rid of the randomness in the integration path by means of a change of a variable [29]. Using a parameter $\epsilon$, the integration path on the right side of (9) does not depend on the random variable anymore. Therefore, it is possible to switch the integration and the expectation operators. In the following cases integrals are solved by means of Gauss quadrature rule: 4 points have been chosen for the surface integration and 3 to 7 points on the uncertain parameter range for the double integration of the probability distribution, depending on the shape of the function and on the required accuracy. The chosen probability distribution has triangular distribution, zero centered, crisp value equal to $1 / a$, lower limit $-a$ and upper limit $a$, and unitary area. Node locations are kept fixed for numerical reasons: this enables easy coupling with FEM velocity for structure models and allows the parametric description of the boundary. This means that the model preserves its shape and only local variability is introduced. The variability in the distance between the field and source points interacts with the wavelength in the high-frequency range and affects the response of the structure.

The introduction of uncertainties in the material, which strongly influences the response of the structure, cannot be taken into account with the current formulation and assumptions. This is mainly due to the fact that the material properties affect all the responses at a nodal location and correlation/decorrelation rules cannot be introduced for them as explained previously.

\section{Application Cases}

In this section we present two academic application cases: a rectangular and a spheric acoustic cavity. Both of them present zero velocity as a condition over the whole boundary and unit monopole inside the cavity. Source intensity is constant with frequency. Models are studied with three different uncertainty values. The cases have, respectively, characteristic value of the uncertain parameter $u$ equal to $0.02,0.05$, and 0.10 scaled to the characteristic dimension of the element (a value of 1 corresponds to the element dimension). The perturbation is supposed to be constant over the whole boundary and source positions. The fluid is air with density equal to $1.3 \mathrm{~kg} / \mathrm{m}^{3}$ and speed of sound equal to $330 \mathrm{~m} / \mathrm{s}$. The solution is evaluated over a field mesh placed in the inner side of the cavity.

The code used is in-house-made and it has been developed in MATLAB.

4.1. Rectangular Acoustic Cavity. This simple application can be useful for instance to model acoustic behaviour of 


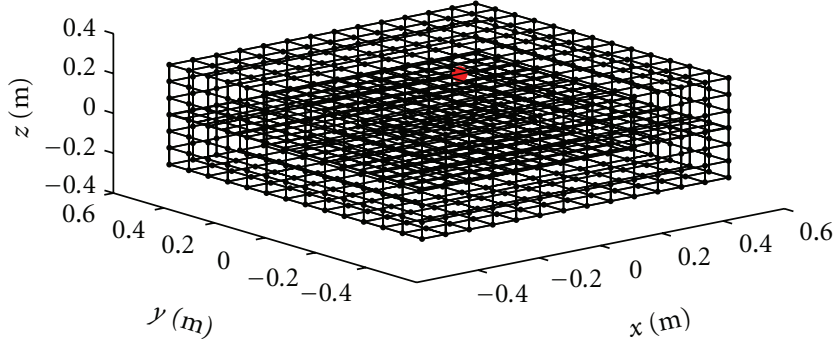

FIGURE 2: Rectangular acoustic cavity, source and field mesh.

TABLE 1: Rectangular acoustic cavity: model characteristics and analysis details.

\begin{tabular}{lc}
\hline Box dimensions & $1 \times 1 \times 0.5 \mathrm{~m}$ \\
Elements & 650 QUAD \\
Nodes & 652 \\
Position of the acoustic source & $(0.2,0.2,0.1) \mathrm{m}$ \\
Characteristic value of the uncertainties, $a$ & $0.02-0.05-0.10$ \\
Frequency range of analysis & $100-3000 \mathrm{~Hz}$ \\
Frequency step & $10 \mathrm{~Hz}$ \\
\hline
\end{tabular}

a room in which a loudspeaker excites the fluid generating wave motion. Walls are modelled as rigid since zero velocity boundary condition is applied.

Model characteristics are specified in Table 1 and its geometry in Figure 2.

It can be immediately noted that 650 elements, with $0.077 \mathrm{~m}$ as main dimension, are not enough to reach $3000 \mathrm{~Hz}$. Indeed, this model can reach about $700 \mathrm{~Hz}$. To correctly simulate up to such a frequency, we need $0.018 \mathrm{~m}$ elements which seriously increase the problem dimension up to around 12000 degrees of freedom.

The field mesh is composed by 441 points, is planar, and is placed at $0.225 \mathrm{~m}$ from the ground. It is rectangular and has the same dimensions of the box. Coordinates of the field solution point are $(-0.0990 \mathrm{~m},-0.3465 \mathrm{~m}, 0 \mathrm{~m}$; the box is centered in the origin).

Figure 3 shows the effect of the uncertainties in the evaluation of Green's functions. Functions with different values of uncertainties are compared with the deterministic ones. Increasing the frequency, the effect of uncertainties starts to smooth the response.

From Figures 4, 5, 6, and 7, it is possible to note the effects of the uncertainties on pressure values over the field point mesh. As the value $u$ increases, the response shape becomes smoother. It is worth noting that in Figure 7 there is an irregularity close to the monopole position. This is a shortcoming of the formulation highlighted in [27], indeed approaching to a sources the SBEM may present some inaccuracies because the assumptions proposed in Section 3.2 are valid as far as the location of the secondary and primary sources are distinct.

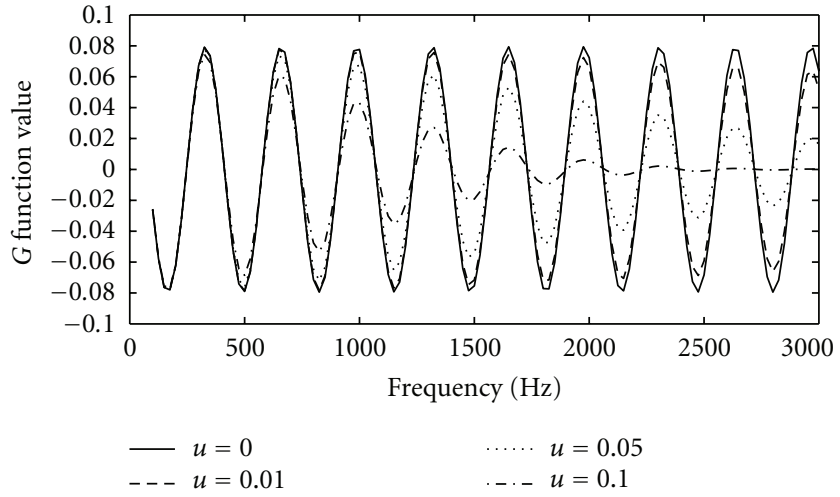

FIGURE 3: Influence of the uncertainties on Green's functions. Three different values are compared with the deterministic one.

Figures 8,9 , and 10 report the response curve at the receiver point. Standard BEM solution, FOM and SOM, obtained with an SBEM, are plotted with three different value of uncertainty. Some considerations can be done. It is clear that using low values of uncertainty BEM and SBEM provides similar results, indeed the curves are almost superposed. Increasing the uncertainty value FOM and SOM have different behaviours. Increasing the frequency, the former vanishes to zero and the latter becomes smoother and asymptotically converges to the smoothed mean value of the deterministic curve which is the response of the nominal system.

Especially, analyzing Figure 10, it is evident that the SOM curve gives a precise representation of the modal behaviour in the low-frequency range. On the other hand, the highfrequency solution is smooth and only delivers information about the general trend.

4.2. Spherical Acoustic Cavity. Model characteristics are specified in Table 2 and its geometry in Figure 11.

Even is this case the mesh is appropriate to accurately predict the acoustic behaviour up to $800 \mathrm{~Hz}$. Results are similar to the ones obtained in the previous case. From Figures 12,13 , and 14, it can be seen that results surface becomes smoother increasing the uncertain parameter $u$. As we already noted, with a high level of uncertainty, if the source is placed near the field mesh, it can produce inaccuracies in the results; see Figure 15.

Analyzing the response functions from Figures 16, 17, and 18 , it is possible to conclude that SOM give an averaged and smooth trend of the prediction. On the other hand FOM vanishes to zero increasing the frequency.

4.3. Discussion. The first set of results presented the results over a field point mesh. Then frequency response functions have been computed at an interior solution point. Observing them is possible to note as FOMs vanish and SOMs become smooth as the frequency increases. Moreover, in the high-frequency range the only contributions that allow to 


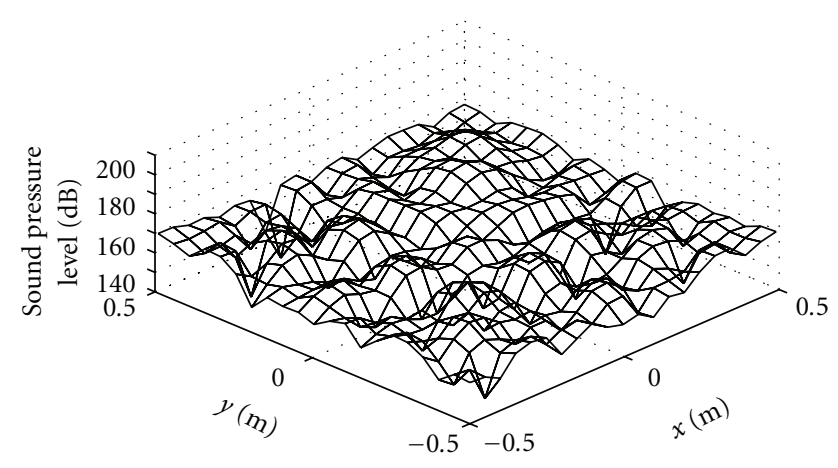

FIgURE 4: Rectangular acoustic cavity. Second-order moments computed in correspondence of the field point mesh at $1200 \mathrm{~Hz}$. No uncertainties are taken into account.

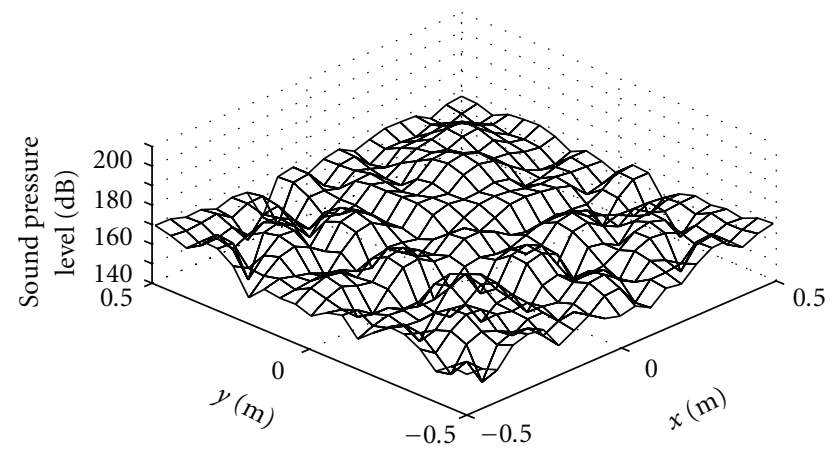

Figure 5: Rectangular acoustic cavity. Second Order Moments computed in correspondence of the field point mesh at $1200 \mathrm{~Hz}$. Uncertainty parameter $u$ is equal to 0.02 .

represent the general trend are the ones that introduce power into the system. Consequently, boundary conditions which do not introduce power do not contribute to the solution and vanish.

It is interesting to compare the presented methodology with the other well-known approaches for vibro-acoustics. A reliable technique to predict the averaged response of a system affected by uncertainties is the Monte Carlo method. Nevertheless, in order to obtain a reliable prediction a large amount of computations is needed, and when models are large, obtaining a result would be very demanding. On the contrary, SBEM allows to avoid the sampling procedure and solve the problem in one go.

If compared with the well-known deterministic techniques, SBEM allows to represent a deterministic behaviour in the low-frequency range. Nevertheless, increasing the frequency, uncertainties start to influence the solution and using those techniques becomes meaningless. Instead, SBEM allows to tackle this problem providing a solution which becomes smoother as function of the perturbations. Nevertheless, the computational effort is increased with respect to the methodologies for the low-frequency range.

We can compare the SBEM formulation with the wellknown methodology for high frequencies. First of all,

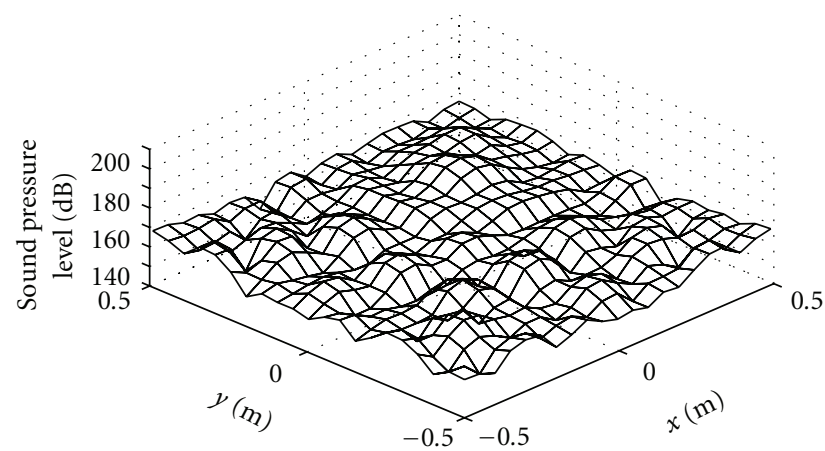

Figure 6: Rectangular acoustic cavity. Second Order Moments computed in correspondence of the field point mesh at $1200 \mathrm{~Hz}$. Uncertainty parameter $u$ is equal to 0.05 .

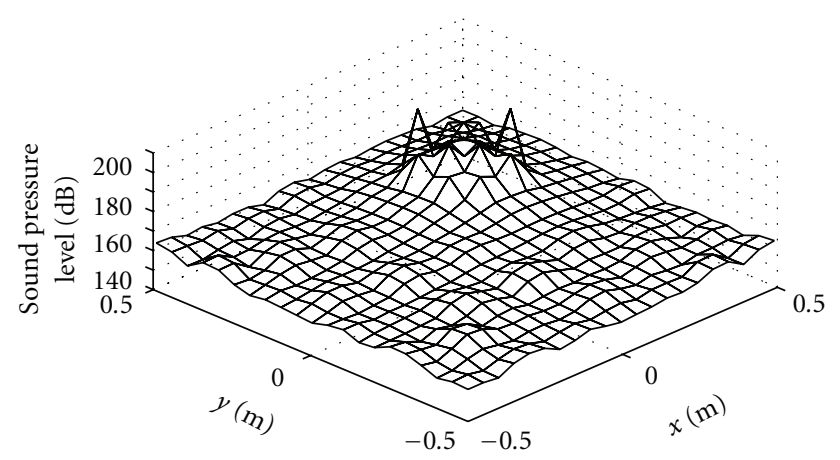

Figure 7: Rectangular acoustic cavity. Second Order Moments computed in correspondence of the field point mesh at $1200 \mathrm{~Hz}$. Uncertainty parameter $u$ is equal to 0.10 .

pulling down SEA weakens its assumptions and consequently the solution may be no longer reliable. On the contrary pushing up the SBEM to SEA validity range may be very computationally demanding. Moreover, SEA allows to obtain a global response, while SBEM provides local details. Finally, SBEM allows a direct control on the geometrical uncertainty of the model shape while SEA does not.

Regarding the applications shown in the previous paragraph, is worth noting that results obtained with classic BEM are not accurate, since the mesh it is not appropriate for the whole frequency range of analysis. Indeed as we previously observed, using SBEM allows a mesh coarsening process. The following examples are carried out using meshes not refined enough to predict the response over such a wide frequency range. Nevertheless, at high frequencies, a coarse model present matrices with high ill-conditioning number. Since resonance is due to matrix ill-conditioning near the eigenfrequency the behaviour of a model with a very coarse mesh is only mathematically similar to a model with high modal density at high frequencies. This can justify an interest in using coarse meshes at high frequencies in order to validate the SBEM. It is also interesting to highlight the relation between the mesh refinement and the degree of uncertainty. With a very coarse mesh the solution is smoothened and 


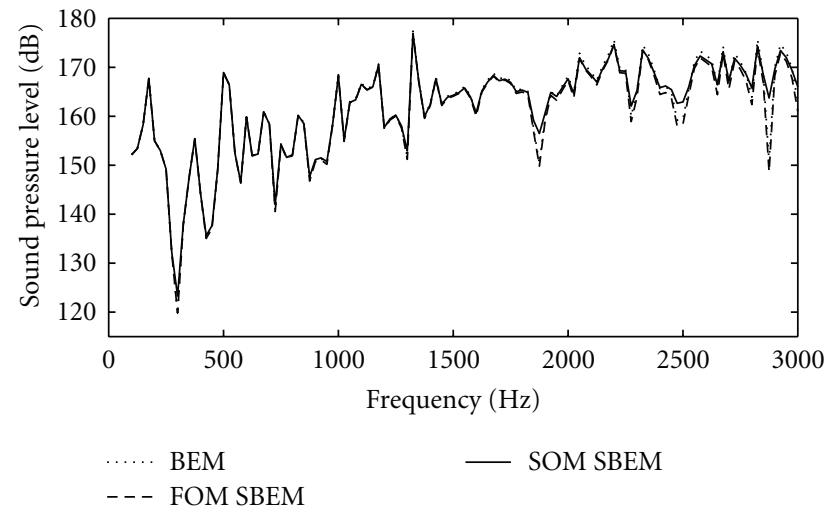

FIgURe 8: Rectangular acoustic cavity. SPL at the solution point $(-0.0990,-0.3465,0)$ : comparison between standard BEM, First Order Moment (FOM) of SBEM and Second Order Moments (SOM) of SBEM. Uncertainty parameter $u$ is equal to 0.02 .

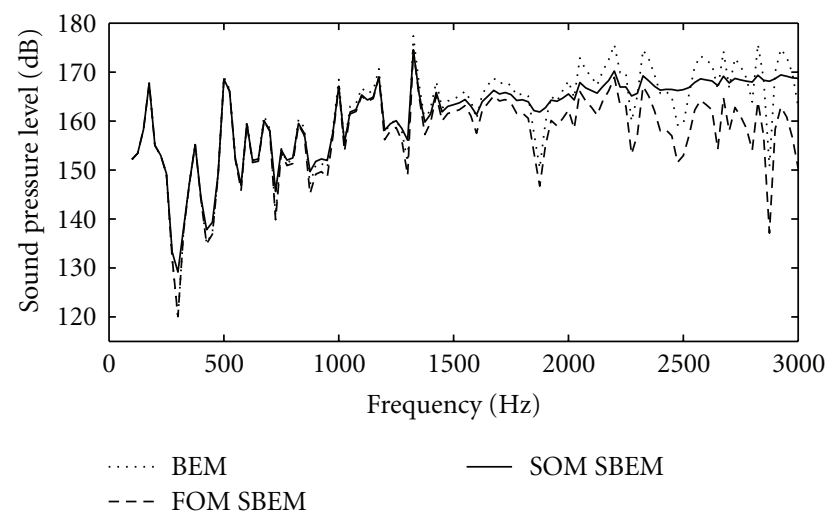

FIgURE 9: Rectangular acoustic cavity. SPL at the solution point $(-0.0990,-0.3465,0)$ : comparison between standard BEM, First Order Moment (FOM) of SBEM and Second Order Moments (SOM) of SBEM. Uncertainty parameter $u$ is equal to 0.05 .

reliable only if the uncertainty degree is high. On the other hand, if perturbations are small, the geometry is almost deterministic and consequently the mesh results inappropriate to predict vibro-acoustic behaviour at high frequencies. A good balance between mesh refinement and degree of uncertainty allows to also reduce the computational effort. Even if the SBEM algorithm has a higher complexity respect to classic BEM, it requires much less elements to predict the smooth trend. Anyhow, matrices are large and complex and handling the problem may be very demanding.

\section{Conclusion}

Predicting structure behaviour of the whole frequency is one of the most appealing objective in vibro-acoustics. Deterministic methods as FEM and BEM are reliable in the low range, but, decreasing the wavelength, small perturbations play a significant role and their use becomes meaningless.

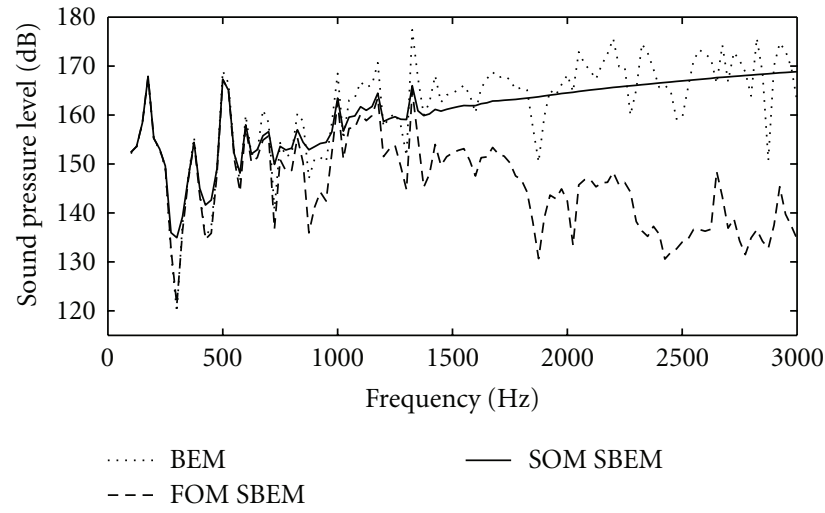

Figure 10: Rectangular acoustic cavity. SPL at the solution point $(-0.0990,-0.3465,0)$ : comparison between standard BEM, First Order Moment (FOM) of SBEM and Second Order Moments (SOM) of SBEM. Uncertainty parameter $u$ is equal to 0.10 .

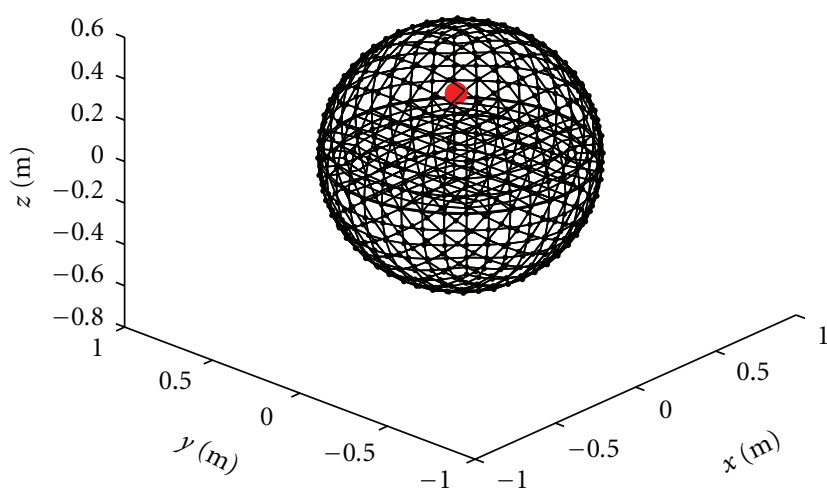

FIGURE 11: Spherical acoustic cavity, source and field mesh.

TABle 2: Spherical acoustic cavity: model characteristics and analysis details.

\begin{tabular}{lc}
\hline Radius of the sphere & $0.6 \mathrm{~m}$ \\
Elements & 600 QUAD \\
Nodes & 602 \\
Position of the acoustic source & $(0.3,0.3,0.1) \mathrm{m}$ \\
Characteristic value of the uncertainties, $a$ & $0.02-0.05-0.10$ \\
Frequency range of analysis & $100-3000 \mathrm{~Hz}$ \\
Frequency step & $10 \mathrm{~Hz}$ \\
\hline
\end{tabular}

The formulations presented in this paper deal with an enhanced BEM approach, the so-called stochastic BEM. Uncertainties are applied to the geometrical properties of the models under proper assumptions. As a result, increasing the frequency of analysis, the prediction becomes smoother and tends to asymptotically converge to the smoothed mean value of the nominal deterministic curve. 


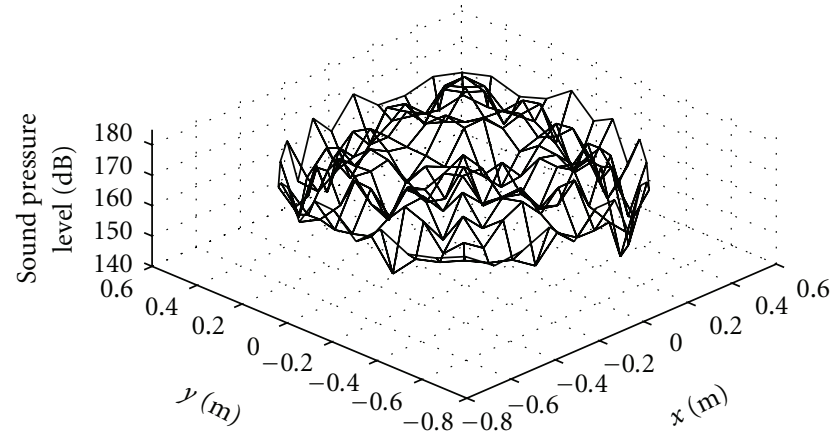

Figure 12: Acoustic spherical cavity. Second-order moments computed in correspondence of the field point mesh at $1200 \mathrm{~Hz}$. No uncertainties are taken into account.

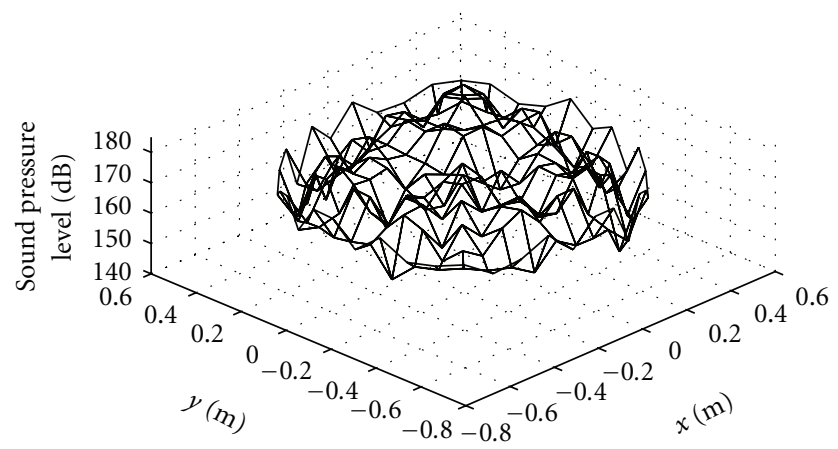

Figure 13: Acoustic spherical cavity. Second-order moments computed in correspondence of the field point mesh at $1200 \mathrm{~Hz}$. Uncertainty parameter $u$ is equal to 0.02 .

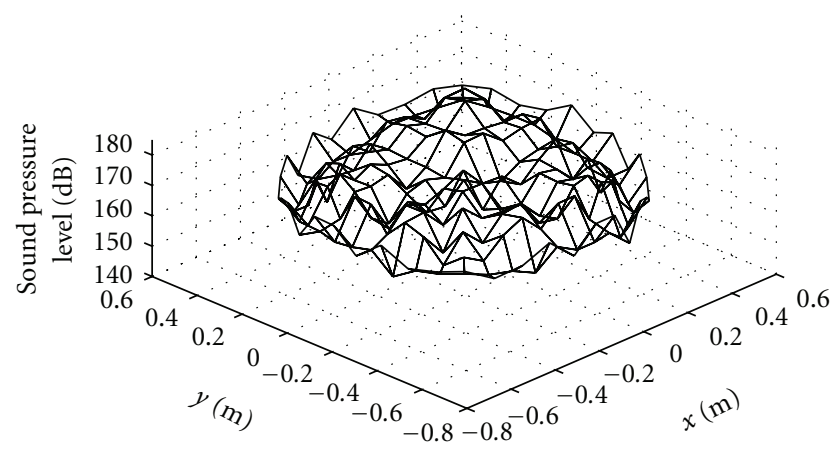

Figure 14: Acoustic spherical cavity. Second-order moments computed in correspondence of the field point mesh at $1200 \mathrm{~Hz}$. Uncertainty parameter $u$ is equal to 0.05 .

SBEM has been applied to two three-dimensional academic cases: a rectangular and a spherical acoustic cavity with zero-velocity boundary condition and a unit source inside. For both of them, the response functions has been studied. Low-frequency behaviour is accurately described and, increasing the frequency, the effects of uncertainties smooth the response and the prediction asymptotically tends

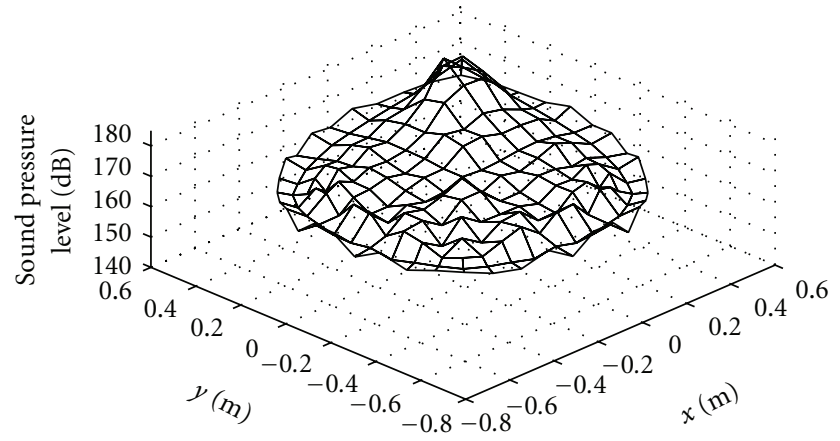

FIgURE 15: Acoustic spherical cavity. Second-order moments computed in correspondence of the field point mesh at $1200 \mathrm{~Hz}$. Uncertainty parameter $u$ is equal to 0.10 .

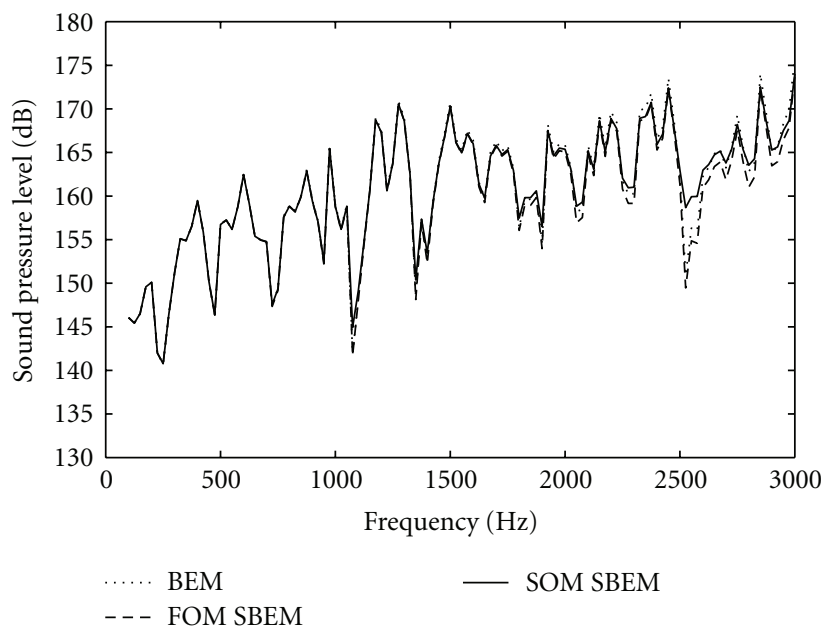

FIgure 16: Acoustic spherical cavity. SPL at the solution point $(-0.2894,0.3726,0)$ : comparison between standard BEM, First Order Moment (FOM) of SBEM and Second-order moments (SOM) of SBEM. Uncertainty parameter $u$ is equal to 0.02 .

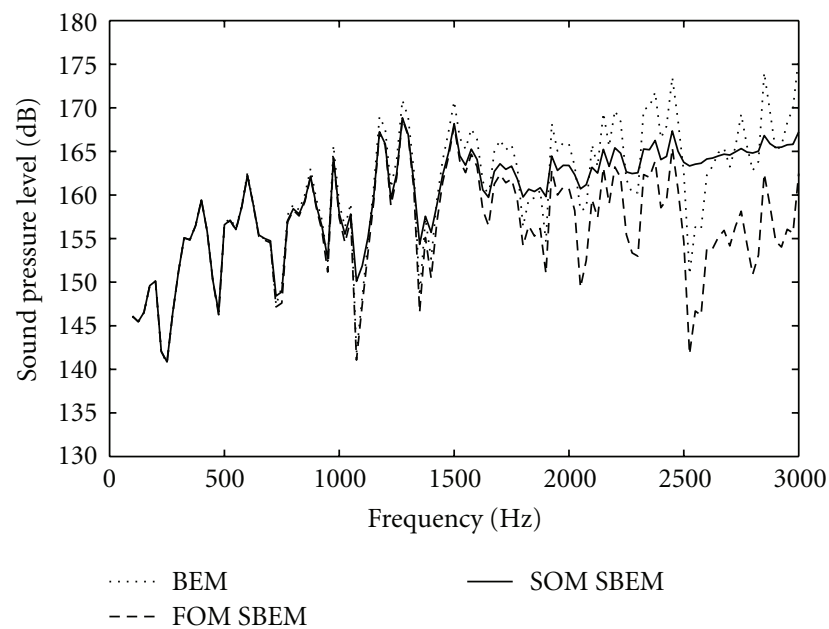

FIgure 17: Acoustic spherical cavity. SPL at the solution point $(-0.2894,0.3726,0)$ : comparison between standard BEM, First Order Moment (FOM) of SBEM and Second-order moments (SOM) of SBEM. Uncertainty parameter $u$ is equal to 0.05 . 


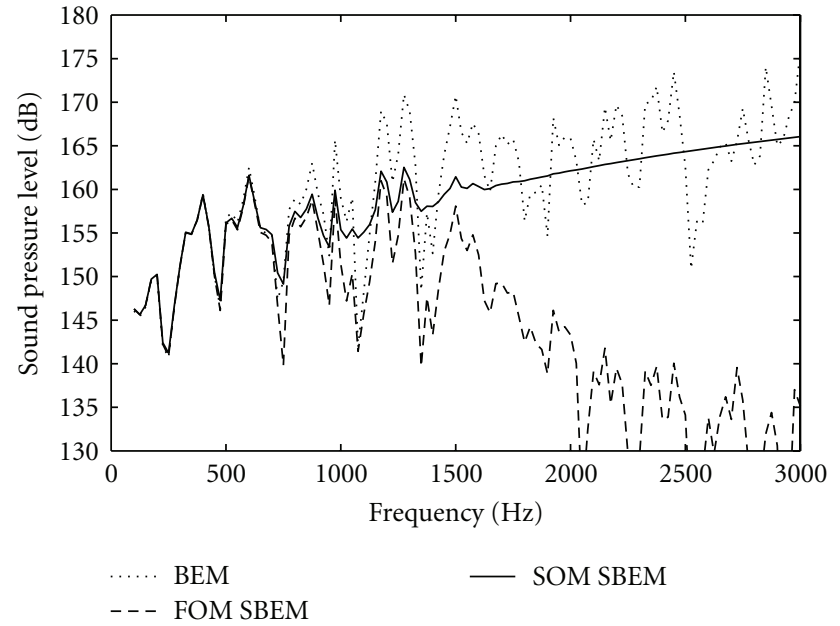

Figure 18: Acoustic spherical cavity. SPL at the solution point $(-0.2894,0.3726,0)$ : comparison between standard BEM, First Order Moment (FOM) of SBEM and Second-order moments (SOM) of SBEM. Uncertainty parameter $u$ is equal to 0.10 .

to the deterministic one. From a computational point of view the effort is decreased in respect to the traditional BEM, thanks to the mesh coarsening process. On the other hand, the number of equations required drastically increases.

Nevertheless, some questions are still open. No rule of thumb exists to correlate the prediction accuracy to the mesh refinement. Moreover, applying a nonzero-velocity boundary condition drastically increases system dimensions because power sources are always connected to the other variables. Research has to be done also in this direction. A computationally less demanding method has to be investigated in order to find a solution to the nonzero problem with reasonable efforts. Finally, up till now, only interior problems have been solved: one of the next step in research is to apply the methodology to exterior cases.

\section{Acknowledgment}

This paper has been cofunded by the European Commission within the MID-MOD project under the 7th Framework Programme (GA-2009-218508).

\section{References}

[1] O. C. Zienkiewicz and R. L. Taylor, The Finite Element Method, vol. 1, 2, McGraw-Hill, London, UK, 4th edition, 1991.

[2] R. Butterfield and K. Bannerjee, Boundary Element Methods in Engineering Science, McGraw-Hill, New York, NY, USA, 1991.

[3] R. D. Ciskowski and C. A. Brebbia, Boundary Element Methods in Acoustics, WIT Press, Southampton, UK, 1991.

[4] R. Hallez and K. De Langhe, "Solving large industrial acoustic models with the Fast Multipole Method," in Proceedings of the International Congress on Sound and Vibration (ICSV'09), Krakow, Poland, July 2009.

[5] H. Briot, M. Tournour, and G. Massa, "On a few recent advances offinite element methods for the Helmholtz equa- tion," in Proceedings of the 16th International Congress on Sound and Vibration (ICSV'09), Krakow, Poland, July 2009.

[6] W. Desmet, A wave based prediction technique for coupled vibro-acoustic analysis, Ph.D. thesis, KU Leuven, Division PMA, Leuven, Belgium, 1998.

[7] C. S. Manohar and A. J. Keane, Statistics of Energy Flows in Spring-Coupled One-Dimensional Systems, MIT Press, Cambridge, UK, 1997.

[8] A. J. Keane and C. S. Manohar, "Energy flow variability in a pair of coupled stochastic rods," Journal of Sound and Vibration, vol. 168, no. 2, pp. 253-284, 1993.

[9] F. J. Fahy and A. D. Mohammed, "A study of uncertainty in applications of sea to coupled beam and plate systems, part I: computational experiments," Journal of Sound and Vibration, vol. 158, no. 1, pp. 45-67, 1992.

[10] N. Baldanzini, "Progettazione meccanica per la riduzione del rumore e delle vibrazioni: lo strumento della Statistical Energy Anlysis," in Proceedings of the 30th Convegno Nazionale Associazione Italiana Analisi delle Sollecitazioni (AIAS '01), Alghero, Italy, Settembre 2001.

[11] R. H. Lyon, Statistical Energy Analysis of Dynamical Systems, MIT Press, Cambridge, Mass, USA, 1975.

[12] R. J. M. Craik, Sound Transmission through Buildings Using Statistical Energy Analysis, Gower, London, UK, 1996.

[13] K. De Langhe, High-frequency vibrations: contribuitons to experimental and computational SEA parameter identification techniques, Ph.D. thesis, KU Leuven, Division PMA, Leuven, Belgium, 1996.

[14] G. Borello and L. Gagliardini., "Virtual SEA: towards an industrial process," in Proceedings of the SAE Noise and Vibration Conference, St. Charles, Ill, USA, 2007.

[15] B. R. Mace and E. Manconi, "Modelling wave propagation in two-dimensional structures using finite element analysis," Journal of Sound and Vibration, vol. 318, no. 4-5, pp. 884-902, 2008.

[16] S. Finnveden and M. Fraggstedt, "Waveguide finite elements for curved structures," Journal of Sound and Vibration, vol. 312, no. 4-5, pp. 644-671, 2008.

[17] P. J. Shorter and R. S. Langley, "Vibro-acoustic analysis of complex systems," Journal of Sound and Vibration, vol. 288, no. 3, pp. 669-699, 2005.

[18] P. Ragnarsson, B. Pluymers, S. Donders, and W. Desmet, "Subcomponent modelling of input parameters for statistical energy analysis by using a wave-based boundary condition," Journal of Sound and Vibration, vol. 329, no. 1, pp. 96-108, 2010.

[19] D. J. Nefske and S. H. Sung, "Power flow finite element analysis of dynamic systems: basic theory and application to beams," in Proceedings of the Statistical Energy Analysis, vol. 3, pp. 47-54.

[20] J. C. Wohlever and R. J. Bernhard, "Mechanical energy flow models of rods and beams," Journal of Sound and Vibration, vol. 153, no. 1, pp. 1-19, 1992.

[21] O. M. Bouthier and R. J. Bernhard, "Simple models of energy flow in vibrating membranes," Journal of Sound and Vibration, vol. 182, no. 1, pp. 129-147, 1995.

[22] O. M. Bouthier and R. J. Bernhard, "Simple models of energy flow in vibrating plates," Journal of Sound and Vibration, vol. 182, no. 1, pp. 149-166, 1995.

[23] Y. Lase, M. N. Ichchou, and L. Jezequel, "Energy flow analysis of bars and beams: theoretical formulations," Journal of Sound and Vibration, vol. 192, no. 1, pp. 281-305, 1996.

[24] M. N. Ichchou, A. Le Bot, and L. Jezequel, "Energy models of one-dimensional, multi-propagative systems," Journal of Sound and Vibration, vol. 201, no. 5, pp. 535-554, 1997. 
[25] M. Viktorovitch, P. Moron, F. Trouverez, and L. Jézéquel, "A stochastic approach of the energy analysis for onedimensional structures," Journal of Sound and Vibration, vol. 216, no. 3, pp. 361-377, 1998.

[26] M. Viktorovitch, F. Thouverez, and L. Jezequel, "A stochastic reformulation of the power flow equations for membranes and plates," Journal of Sound and Vibration, vol. 211, no. 5, pp. 910-917, 1998.

[27] M. Viktorovitch, F. Thouverez, and L. Jezequel, "A new random boundary element formulation applied to high frequency phenomena," Journal of Sound and Vibration, vol. 223, no. 2, pp. 273-296, 1999.

[28] M. Viktorovitch, F. Thouverez, and L. Jezequel, "An integral formulation with random parameters adapted to the study of the vibrational behaviour of structures in the middle- and high-frequency field," Journal of Sound and Vibration, vol. 247, no. 3, pp. 431-452, 2001.

[29] A. Pratellesi, Noise and vibration analysis in the mid frequency range, Ph.D. thesis, Università degli Studi di Firenze, Firenze, Italy, 2007.

[30] A. Pratellesi, M. Viktorovitch, N. Baldanzini, and M. Pierini, "A hybrid formulation for mid-frequency analysis of assembled structures," Journal of Sound and Vibration, vol. 309, no. 3-5, pp. 545-568, 2008.

[31] M. Viktorovitch and A. Pratellesi, "A hybrid mid-frequency formulation for vibro-acoustic predictions," Noise Control Engineering Journal, vol. 56, no. 1, pp. 71-84, 2008. 

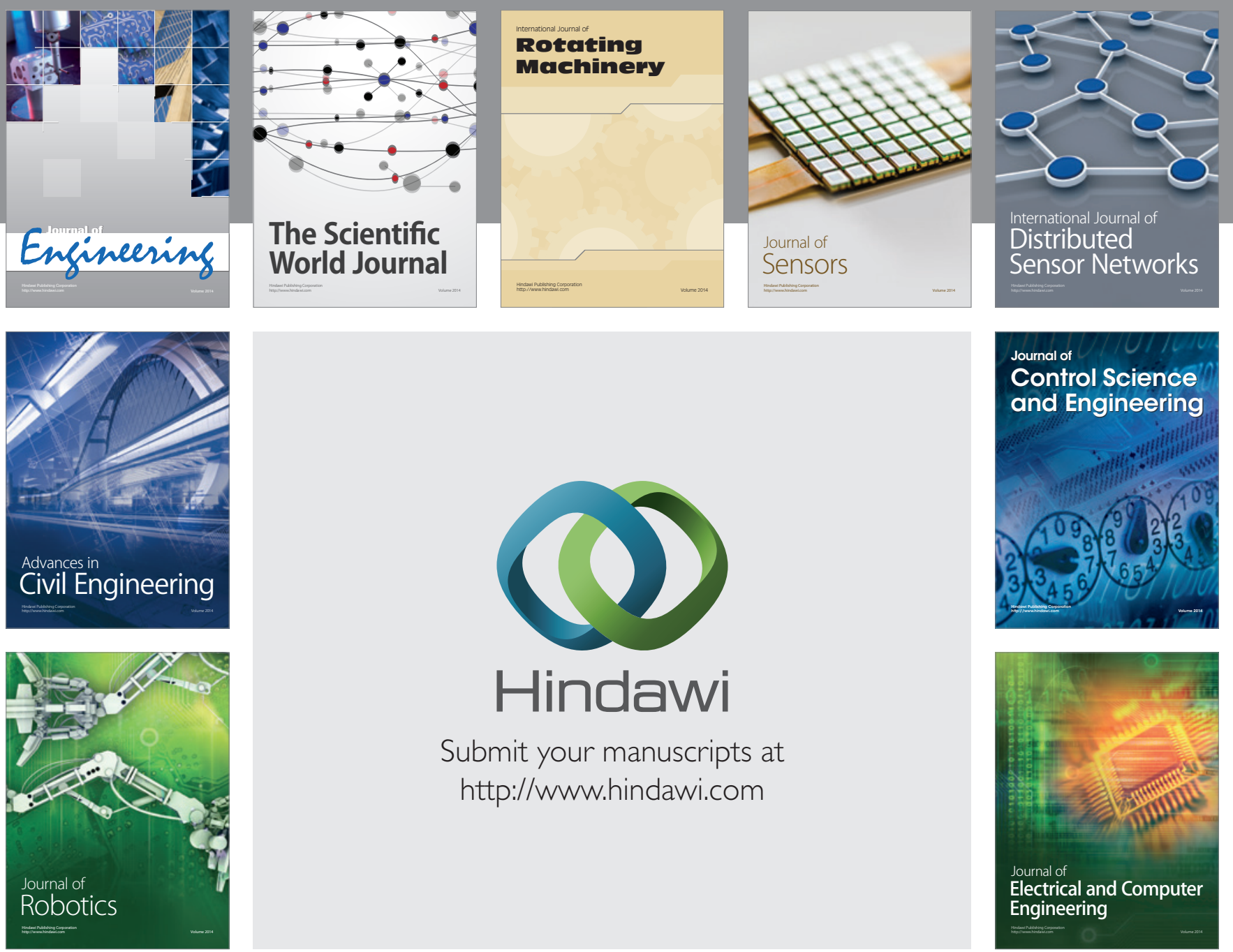

Submit your manuscripts at

http://www.hindawi.com
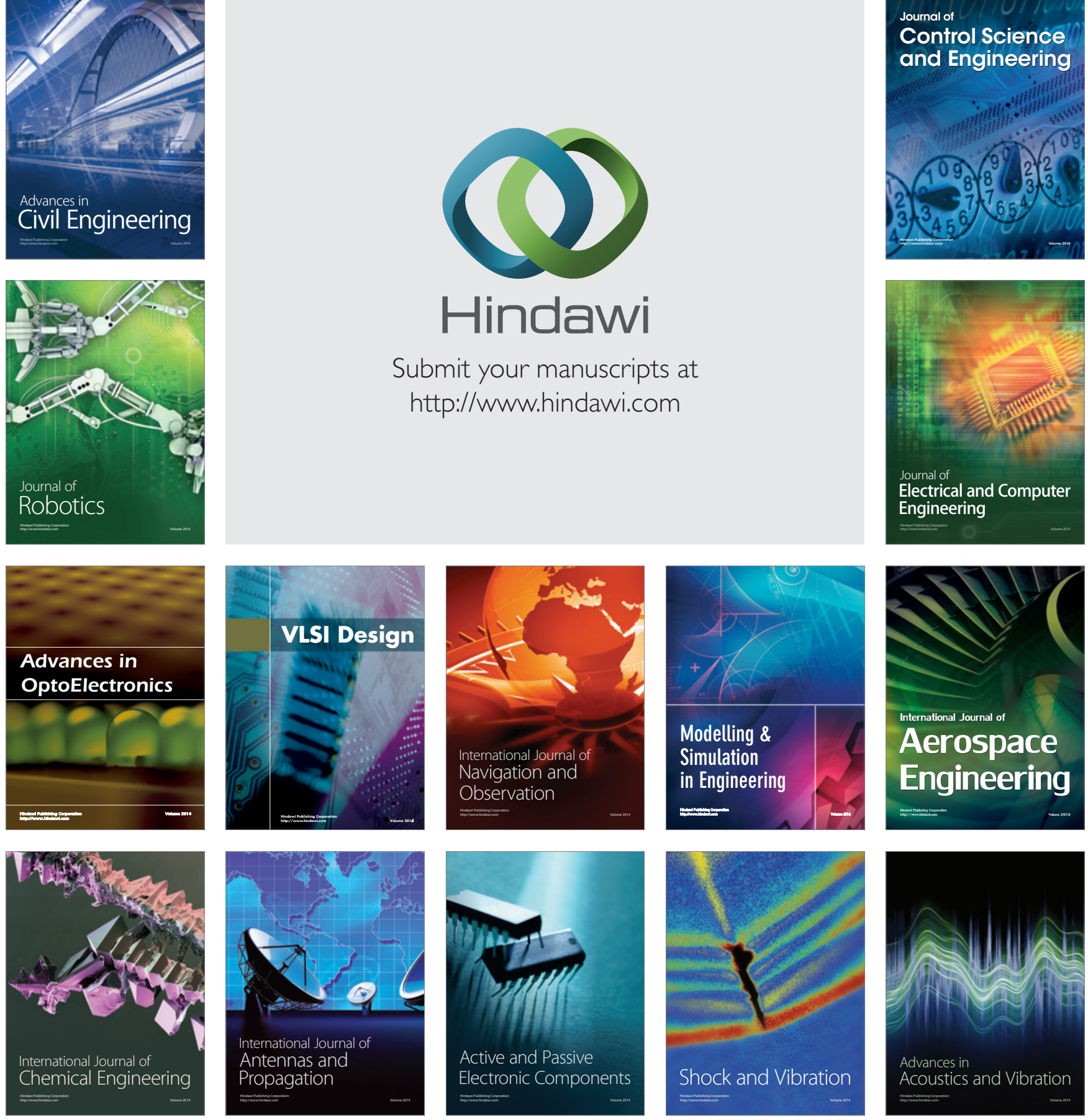